\title{
La historia urbana en Francia: veinte años de investigaciones*
}

\author{
Bernard Lepetit \\ ÉCOLE DE HAUTES ÉTUDES \\ EN SCIENCES SOCIALES
}

$\mathrm{U}$ no de los primeros balances de la investigación francesa sobre historia urbana estaba dirigido a un público británico. La historia urbana inglesa, que en los años sesenta pregonaba su voluntad de constituirse en disciplina autónoma, deseaba disponer de un balance comparativo, quizás, para medir el camino que ella misma había recorrido. Es fácil imaginar la dificultad del autor para delimitar un campo que entonces no tenía existencia propia en Francia. Su conclusión fue muy pesimista

Si consideramos los resultados alcanzados por la historia urbana en Francia durante los últimos cincuenta años, debe. mos admitir que la cosecha es magra y

* Traducción: Gabriela Montes de Oca. más bien decepcionante. Incluso ahora, este campo de investigación histórica está aún en su infancia. ${ }^{1}$

En la actualidad, el paisaje parece radicalmente transformado; la historia urbana es una obra en construcción en la que se trabaja con empeño. Desde hace veinte años se acumulan las grandes tesis de doctorado. ${ }^{2}$ La rápida sucesión de coloquios, la cantidad de participantes que movilizan, las frecuentes publicaciones que se llevan a cabo después, dan testimonio de tal

1 Bedarida, "Growth", 1968.

2 Goubert, Beauvais, 1960; Deyon, Amiens, 1967; Garden, Lyon, 1970; Perrot, Genèse, 1975; Bardet, Rouen, 1983; Jacquemet, Belleville, 1984. S6́lo cito los trabajos que conllevan una ambición de historia total, desarrollada en un marco monográfico. 
vitalidad. ${ }^{3}$ La colección titulada Historia de las Ciudades, fundada en 1972 y destinada a un público heterogéneo de aficionados locales y profesionales de la historia, es un éxito de librería. Por último, Historia de la Francia ur. bana, un panorama en cinco volúmenes y más de tres mil páginas, según mi entender no tiene equivalente en el extranjero. ${ }^{4}$

Sin embargo aunque encontramos, por ejemplo, cátedras de historia de América Latina o de historia de las relaciones internacionales en distintos centros universitarios, en vano buscaríamos una cátedra sobre la historia de las ciudades. Fuera de la universidad no existe otra institución de aglutinación que esté bien estructurada. El grupo de Trabajo Internacional de Historia Urbana, creado en 1976 en el marco de la Maison des Sciences de l'homme (Casa de las Ciencias del Hombre) en París y en el cual los investigadores franceses siempre han desempeñado un papel importante, sigue siendo muy informal. Ninguna revista especializada puede además pretender cumplir le. función de abanderada o de espacio de intercambio intelectual. Remitir esta situación de hecho al habitual individualismo de los investigadores es una explicación demasiado breve, podría valer para to-

${ }^{3}$ Algunas publicaciones recientes: Livet, $P$ ouvoir, 1983; Garden, Construire, 1984; Lepetit, Ville, 1987; Magri, Villes, 1989; Biget, Cadastres, 1989.

"La colección "Histories des villes de France" es publicada por el editor E. Privat en Toulouse; cada ciudad es objeto de una monografía (han publicado alrededor de 40), estudiada desde la antigüedad hasta nuestros dias. En 5 vols., y bajo la dirección de Duby, Histoire, 1980-1985. dos los sectores de la historia, incluso para los más definidos. ¿Cómo explicar entonces la situación de la historia urbana en Francia? Es necesario precisar la orientación y el contenido de los trabajos correspondientes. Pero sin duda, eso no basta para comprender un campo nuevo que, al mismo tiempo, es abundante y está poco estructurado. Sus características actuales y su porvenir cercano dependen, tanto de las condiciones de su formación como de su lugar en la geografía del saber. Ésas serán las principales etapas de este análisis.

Antes de comenzar, debo indicar los límites del estudio. La gran cantidad de trabajos y el espacio necesariamente restringido de un artículo, impiden citar todo. Mi propia especialización y la inevitable subjetividad ocasionan una arbitrariedad en la elección. Los ejemplos corresponderán casi siempre a lo que se denomina historia moderna, del siglo XVI al siglo XVIII. Quizás estos límites cronológicos no desequilibren demasiado el cuadro, dado que el análisis de la ciudad preindustrial ha originado buena parte de la renovación de la historia urbana.

\section{LAS CONDICIONES DE LA FORMACIÓN DE LA HISTORIA URBANA}

Cuando en 1970 Annales consagra un número especial al tema historia y urbanización, ubica claramente la tarea:

Una vez más queremos invitar a la reflexión histórica sobre un problema 


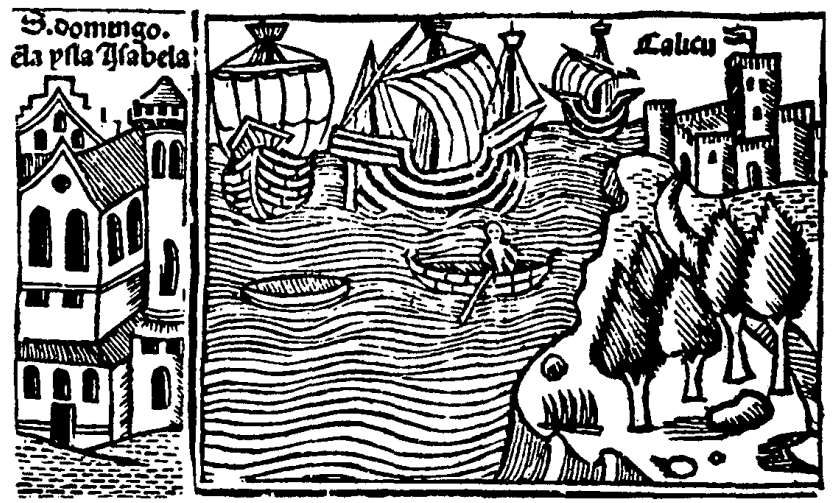

muy actual. El desarrollo urbano moviliza desde hace varios años todas las ciencias humanas, no sólo para imaginar proyectos de acondicionamiento, remedios para la extraordinaria patología que se ha engendrado, sino de un modo más profundo para encargarse consciente y científicamente de lo que parece ser el problema esencial de nuestras sociedades. En este concierto colosal el historiador tiene, desde luego, algo que decir.5

Todo o casi todo está dicho en una frase: lo social resumido en lo urbano, el desarrollo de las ciudades y el subsiguiente desorden, el acondicionamiento necesario, la movilización general de las ciencias humanas y la intervención de la historia. Expectativa social, clima de requerimiento

5 "Histoire", 1970. científico no exento de rivalidades entre las disciplinas; nos esforzaremos por sugerir que, a dichas solicitudes externas, se añade una necesidad interna de la historia, ligada a su evolución.

\section{La demanda social}

A menudo se ha subrayado la coincidencia entre los grandes momentos de desarrollo de la historiografía y los periodos de crisis urbana. ${ }^{6}$ Del siglo XVI al XVII, del humanismo a la ilustración, congregaciones religiosas y notables eruditos multiplican las historias de las ciudades. La exaltación de la civilización citadina, la investigación sistemática de los signos

6 Roche, "Urban", 1980, pp. 12-22. 
de grandeza (vestigios arqueológicos, grandes hombres, fastos de los perioclos de independencia de la ciudad) en un pasado que cauciona permanentemente al presente, constituyen los temas esenciales de esta literatura. Su ambición es evidente. En un largo periodo de ataque contra la independencia de las ciudades, durante el cual las antiguas autonomías locales retroceden ante el poder real, se trata de valorar culturalmente los vestigios de una independencia perdida. Durante todo el siglo XIX, tres generaciones de eruditos locales, impulsores de asociaciones culturales, se dedican a su vez a redactar historias urbanas. En éstas se manifiesta una voluntad de arraigamiento en el pasado cuando la industrialización llega a romper los antiguos equilibrios, a provocar el crecimiento de las ciudades y se apela de nuevo a las jerarquías urbanas, cuando el ciclo hausmanniano de los acondicionamientos afecta al tejido mismo de la ciudad. Se sostiene una indepen. dencia exagerada en un momento en que, bajo el efecto del desarrollo de los transportes y de los cambios en la orientación de las inversiones, se unifica el espacio económico nacional.

El florecimiento historiográfico actual se inscribe también en un movimiento de profunda transformación urbana. En un país que durante largo tiempo siguió siendo más campesino que muchos de sus vecinos, el cambio cuantitativo es considerable: en 1950, el $55 \%$ de los franceses vivían en las ciudades; en la actualidad es el $85 \%$. La dinámica de la urbanización generalizada toma rasgos de recuperación, y la ciudad necesariamente se vuelve objeto de una nueva atención. La interrogación es tanto más ansiosa cuanto que el crecimiento se acompaña de mutaciones cualitativas evidentes. Con la afluencia de nuevos habitantes, las ciudades han tenido que recomponer su espacio, enfrentarse a graves problemas sociales, afrontar los múltiples efectos negativos del desarrollo. La expectativa social, frente a un fenómeno que parece ineluctable e incontrolable, se explica en dos niveles y de dos diferentes maneras.

Lo más general es también lo más difuso. En una sociedad francesa ligada a la tierra y cuyo ideal urbano estaba bastante bien representado en las ciudades pequeñas, ${ }^{7}$ el rápido movimiento de urbanización y, de manera măs general, el crecimiento económico, constituyeron un desarraigo. A modo de compensación nostálgica se desarrolla un deseo de arraigamiento. El desarrollo del fenómeno de la residencia secundaria rural, el entusiasmo por la reconstitución genealógica familiar, el interés por la historia de un "mundo que hemos perdido" son ejemplos de ello. La investigación urbana se incluye en esta corriente, que de esta manera desborda en gran medida los límites de la profesión. La demanda de estado obedece evidentemente a razones distintas: se trata aquí de comprender para prever y organizar. La importancia del impulso administrativo en el desarrollo de la investigación urbana ha sido considerable. Institucio-

7 "Lo que acaba de ser vencido en nosotros, es precisamente nuestra querida ciudad pequeńa", escribía Marc Bloch en 1940 . Bloch, $L^{\prime}$ étrange, 1946 (reedición de 1990). 
nes como el Centro Nacional de Investigación Científica, la Delegación General de Investigación Científica y Técnica o la Dirección de Arquitectura y la Comisión de Investigación Urbana del Ministerio de Urbanismo y Vivienda destinaron medios financieros para sostener equipos y llevar a cabo programas de investigación. No se encuentra necesariamente un esfuerzo equivalente en otros sectores de las ciencias humanas.

Los historiadores no fueron los primeros invitados a constituir estos equipos. Ia fe tecnocrática en las posibilidades de un acondicionamiento funcional, además de la esperanza de descubrir la teoría general de la urbanización susceptible de regir el problema urbano fueron al principio más importantes. El fracaso condujo a una doble reorientación de las investigaciones. Por una parte, las indagaciones se dirigieron mucho más hacia las situaciones particulares de sociabilidad y multiplicidad de las formas de vida locales. Por otra parte, con frecuencia manifestaron una voluntad de ampliación cronológica. Había tres razones que abogaban por tomar en cuenta el tiempo:

1) La ciudad, en las zonas de Europa occidental con gran densidad de población y una urbanización antigua, es un fenómeno de muy larga duración. La débil maleabilidad de las formas urbanas se suma a la permanencia de los lugares para garantizar al pasado el peso máximo. La ciudad es un conservatorio temporal. ${ }^{8}$ 2) La ciudad es un fenómeno total que mezcla todos

8 Perrot, Genèse, 1975, p. 553. los niveles de la realidad: económico, social, político, cultural. Por ello, todo cuestionamiento fraccionado, de la sociología o de la ciencia económica, por ejemplo, se expone a carecer de perti. nencia. Por el contrario, en una lectura totalizante, como afirma serlo el análisis histórico, se puede tomar en cuenta mejor el efecto de las interrelaciones. 3) Dos tradiciones recorren el urbanismo: un modelo progresista nutrido de lógica funcional y otro, culturalista, preocupado por encontrar la antigua unidad orgánica de la ciudad.$^{9}$ La percepción de los límites de una adecuación tecnocrática incapaz de resolver los problemas urbanos no podría dejar de valorar la segunda corriente. El pasado, entonces, conserva el testimonio de los modelos logrados, aunque en el orden de lo mítico. A los historiadores toca redescubrirla.

\section{La geografía del saber}

Desarrollada tardíamente, la historia urbana se enfrenta a los análisis realizados por las ciencias sociales vecinas. Los geógrafos, los economistas, los sociólogos, los arquitectos, los filósofos y otros especialistas se han pronunciado acerca de la ciudad. Así, ésta se encuentra expuesta a los cuatro vientos de la investigación y se enfrenta a las grandes corrientes que la impulsan. Para describirla, es necesario medir también ese campo de fuerzas.

El estudio histórico de las funciones citadinas se alimentó en un principio de los métodos y de las reflexio-

\footnotetext{
9 Choay, I' urbanisme, 1965.
} 
nes de los geógrafos. Desarrollados en el marco de las investigaciones universitarias o de las relaciones destinadas a guiar la acción de los organismos de acondicionamiento del territorio, estos análisis desarrollaban una forma de proceder en dos tiempos para comprencler el crecimiento de las ciudades y las configuraciones de la armazón urbana. Se trataba de clasificar primero las ciudades, según el nivel y el tipo de las funciones que ejercían cada una para proceder a continuación, con la ayuda de diversas combinaciones, a la realización de tipologías y jerarquías urbanas. ${ }^{10}$ Por lo menos, tres razones facilitaron la introducción en la historia de ese tipo de interrogantes: una frecuente formación en común, porque la historia y la geografia constituyeron durante mucho tiempo disciplinas asociadas en las cátedras universitarias; un método descriptivo de las actividades económicas; una gran atención de la mayoría de estos estudios geográficos a un pasado, en el cual el presente encontraba su explicación. Más allá de la dimensión descriptiva de las funciones urbanas, los historiadores retuvieron de ella una serie de problemas sobre el modo de funcionamiento económico de las ciu. dades y sobre la naturaleza original de las armazones urbanas de la época preindustrial.

En to que se refiere a las formas urbanas, se proponían al historiador tres modelos de lectura, paralelos al suyo propio. El primero, de inspi-

\footnotetext{
${ }^{10}$ A modo de ejemplo: Dugrand, villes, 1963. Para un análisis crítico: Pumain, "Fonctions", 1976, pp. 385-440.
}

ración marxista, se desarrollaba como reacción a las certidumbres de los responsables técnicos del urbanismo: al becho urbano sucedia la cuestión urbana. ${ }^{11}$ Allí donde los planificadores veían en las formas que tomaba el desarrollo de las ciudades un desorden al que una política adecuada pondría remedio, los neomarxistas veían, por el contrario, la expresión de un orden, el del capital, servido por la política, la del Estado. Así pues, las disparidades vinculadas al crecimiento ya no son accidentales sino consecuencia de determinantes económicas y políticas que es necesario descubrir. ${ }^{12}$ Es difícil apreciar la influencia de esta corriente de pensamiento en la historia urbana.

Se pueden descubrir orientaciones comunes sin dificultad: la preocupación por incluir lo urbano en la sociedad abarcadora o el análisis de la construcción del espacio citadino en términos de problema social. Pero a la inversa, la atención que pone el neomarxismo en las sociedades capitalistas desarrolladas; la absoluta prioridad que otorga a la elaboración teórica sobre la observación empírica; y por último, la disolución que lleva a cabo del problema urbano en el problema social, disolución que impide pensar en la autonomía de lo urbano y en la especificidad del campo de la investigación, constituyen numerosos obstáculos a la comunicación con los historiadores. Éstos, sin duda, sólo en-

11 Carrière, Fait, 1963; Castells, Question, 1972.

12 Además de $M$. Castells, referirse a Lefevbre, Révolution, 1970; Lefevbre, Pensée, 1972; Lojkine, Marxisme, 1977. 
contraron en el conjunto de dichos trabajos lo que la difusa influencia marxista permitía buscar respecto a la historia francesa.

Mucho más directa era la interpelación procedente de un grupo de arquitectos y de urbanistas y de una corriente que se nutría del pensamiento de Michel Foucault. Aquí, en el momento en que la historia se volvía urbana, la investigación urbana se hacía histórica. La parte que le corresponde a la historia urbana stricto sensu se torna difícil de apreciar en las investigaciones a menudo realizadas en el momento mismo. De los trabajos llevados a cabo, en particular en el marco del Instituto Francés de Arquitectura, pueden extraerse grandes interrogantes: ${ }^{13}$ iqué puede decir un proyecto urbano sobre la sociedad en la que se produce? ¿Qué relaciones se establecen entre las representaciones mentales, los planes de acondicionamiento y las operaciones realizadas? ¿Cuáles son las restricciones formales heredadas del pasado que pesan sobre el acondicionamiento urbano? ¿Cómo utiliza una sociedad los edificios para darles un nuevo sentido? La atención dada más a lo repetitivo que a lo excepcional, a lo habitado más que a lo monumental, a la diversidad de lo vivido tras las reglas urbanísticas, a la clialéctica pasado-presente (un presente siempre sometido al peso del pasado, pero que siempre vuelve $a$ investirlo de valores nuevos) constituyen más temas de interés para la cor-

13 Véanse por ejemplo Boudon, Système, 1977; Demangeon, Vaisseaux, 1978; Castex, Lecture, 1980. poración histórica en cuyo seno se desarrollan interrogantes similares.

La lógica de recurrir a la historia nace para la investigación arquitectónica a partir del objeto mismo del análisis: la ciudad tiene una densidad temporal. Para Michel Foucault, la razón de la atención al pasado es otra. Ésta se origina en un funcionalismo revisado. Históricamente, sobre todo en los siglos XVII y XVIII, la ciudad es para él, el lugar y el medio deí reforzamiento del poder. A través de las reflexiones del filósofo y de los equipos que anima o inspira, la ciudad aparece como el lugar privilegiado de aplicación de métodos para disciplinar a las clases inferiores. Esta aplicación se lleva a cabo, para el mayor benefi cio del orden social y de la productividad del trabajo, en favor de las clases dirigentes. Se trata de una visión de la ciudad bastante instrumental; en efecto, es al mismo tiempo la multiplicación de los instrumentos de control social (prisiones, hospitales) y el ordenamiento del espacio público (ampliación del alumbrado de las calles, numeración de las casas) que participan en este sometimiento más eficaz. ${ }^{14}$ De espacio de libertad, la ciudad se transforma en instrumento de poder. Este cambio de las funciones urbanas, menos fácilmente mensurable que el de los geógrafos o los economistas, puede fecharse con exactitud. Así, el papel histórico de la ciudad, organizada y disciplinaria, es lo que lleva a Foucault a econtrar a los historiadores en su terreno. El pueblo y las policías,

14 Foucault, Surveiller, 1975 y Macbines, 1976; Farge, Viere, 1979. 
objeto de una atención renovada, coinciden en el espacio citadino; una vez más se vinculan aquí relaciones sociales, políticas urbanas y organización de territorio.

\section{La evolución de la disciplina bistórica}

El mimetismo, o la moda de la época, no bastan por sí solos para explicar la atención de la historia por lo urbano. De igual modo deben encontrarse razones dentro de la disciplina misma. En primer lugar, se constatará que la historia urbana se integra perfectamente a esta nueva bistoria, cuya práctica se ha casi generalizado gracias a la difusión de la influencia de la Escuela de los Anales:

Para delimitar la ambición y el desarrollo, [lo más simple] es mantener juntos dos objetivos y dos ideas: que la historia se enriquezca con sujetos y métodos al recurrir a otras disciplinas vecinas $e$ incluso con la fragmentación provisional de los compartimentos disciplinarios; y que, sin embargo quede un saber global, ecuménico, que reúna las condiciones de inteligibilidad máxima de los fenómenos sociales. 15

¿Recurrir a las disciplinas vecinas, eliminación provisional de las divisiones? Basta con consultar el índice de todas las primeras grandes tesis de historia urbana, para constatar cómo se perfila ahí la ambición: condiciones demográficas, actividades económicas,

15 Furet, L'Atelier, 1982, p. 9. sociedad urbana, vida religiosa y poder, por aquí; el espacio de los hombres, la economía y sus ritmos, el estilo de vida, por allá; la demografía, estructuras sociales, estructuras mentales y comportamientos colectivos, acullá.

Se verá ahí otra razón de su éxito. En el momento en que la ambición originaria por la historia total retrocede para convertirse en horizonte de investigación, $o$ incluso se borra totalmente, el desarrollo de la historia urbana puede aparecer como un paliativo. He aquí en efecto un objeto de estudio, la ciudad, que "agrupa todas las preguntas planteadas por la evolución plurisecular del sistema de civilización". ${ }^{16}$ ¿Cómo no esperar de su descripción, o mejor aún de su comprensión, un nuevo acercamiento a la globalidad? Si toda la historia se encuentra en lo urbano, comprender la ciudad se convierte en un medio para reconquistar el paraíso perdido y para tener acceso a un saber total renovado.

Vamos a plantear una hipótesis más, menos globalizante, que se ilustrará con un ejemplo, el de la evolución de la historia social en Francia. Durante quince años, el debate al respecto ha girado en torno a una pregunta. ¿Cuáles son las categorías pertinentes para comprender la organización de una sociedad y, en particular, la de la sociedad preindustrial? Ernest Labrousse y sus alumnos propusieron definir primeramente las estructuras sociales en términos de la categoría socioprofesional (en forma de códigos elaborados para el Insti-

${ }^{16}$ Roche, "Ville", en Le Goff, Nowvelle, 1978, pp. 565-570. 
tuto Nacional de Estadística y de Esrudios Económicos para la Francia Actual) y la jerarquía de las fortunas (medida gracias a un análisis de serie de los archivos fiscales y notariales). La principal oposición se originó en un grupo de historiadores inspirados por Roland Mousnier. Para ellos, la sociedad del antiguo régimen no era una sociedad de clases, sino de órdenes. Además en él la jerarquía social no podía deducirse de una escala de fortunas o del lugar de cada uno en las relaciones de producción. Por el contrario, se basaba en la estima social colectiva atribuida a cada situación o a cada función y la única división aceptable era la que reunía a los grupos que hacían que sus hijos contrajeran matrimonio entre ellos.

Entre estos a priori irreductibles, es evidente que no era posible diálogo alguno. Después de una fase de movilización de ambas partes, que originó buenos trabajos (entre ellos las primeras tesis de historia urbana), se llegó a un punto muerto total y esterilizante. Para reanudar la investigación, fue preciso abandonar este terreno de lucha sin salida. Jean-Claude Perrot aportó las primeras justificaciones para tal actitud en un artículo con un título revelador:

Relaciones sociales y ciudades. Se leen ahí tres proposiciones concatenadas: los estudios estructurales, cuando constituyen un fin en sí mismos, encierran a la historia en un debate dogmático; el análisis de las relaciones sociales, y ya no de las estructuras, constituye el medio de superar la esterilidad de este debate; el estudio de los hechos urbanos es una forma privilegiada de actualizar dichas relaciones, pues el espacio urbano consagra las relaciones urbanas. ${ }^{17}$

He aquí la historia urbana, que es considerada como la vía para salir de un estancamiento historiográfico específico, mediante la superación de los bloques de la historia social.

\section{LA NUEVA HISTORIA URBANA}

\section{La invención de lo urbano}

Para precisar la transformación cualitativa que ha acompañado al desarrollo cuantitativo de la historia urbana, sin duda serán suficientes dos textos publicados con quince años de intervalo cada uno. Su carácter tan revelador nos autoriza quizás a citarlos ampliamente. En primer lugar, veamos cómo Pierre Deyon en 1967 justificaba una de las primeras monografías urbanas realizadas en Francia en fecha reciente.

Nuestro conocimiento del siglo XVII incluye muchas sombras; más de un problema ha quedado en suspenso, lo que suscita la curiosidad y las discusiones de los historiadores. El estudio minucioso de la sociedad en la época clásica aparece como una de las condiciones necesarias para la realización fructífera de estos debates; el ensayo que sigue pretendería ser una contribución a esta empresa colectiva. El marco local imponía límites, pero sólo éste permitía medir, contar, realizar acercamientos con-

17 Perrot, "Rapports", 1968, pp. 241-268. Aquí se encuentran las referencias anteriores que permiten seguir el debate. 
tinuos entre los distintos planos de la realidad histórica y tal vez descubrir las relaciones nuevas que otros estudios podrían retomar y verificar. Amiens, capital provinciana: es el estado de las fuentes que, en una gran parte, determinaron esa elección geográfica. Como centro importante de comercio y de fabricación textil, Amiens no constituía, desde el punto de vista económico, un observatorio desdeñable. En todo caso, ofrecía un marco cómodo para un estudio de los ascensos y las diferenciaciones burguesas. ${ }^{18}$

En ese momento la historia urbana aparece muy claramente como un medio y no como un fin. Al encontrar su justificación fuera de ella misma, en realiclad no ha alcanzado su autonomía. Se sitúa en una historia social que la abarca y se plantea por ambición contribuir a los debates que la inspiran. ¿Por qué entonces la ciudad? Esencialmente porque constituye un cómodo marco, un observatorio privilegiado. Como mundo de contrastes sociales, donde las jerarquías se afirman de manera más estructurada y al mismo tiempo sutil, proporciona un campo privilegiado para las controversias de la historia social. Es lugar de ejercicio de un poder administrativo que se dedica al control cuantificado de los hombres y de los productos, un espacio de acumulación archivístico. Por último, es el microcosmos donde se reúnen todos los planos de la realidad, donde se observan realidades explicativas que de otro modo no se percibirían por estar demasido dispersas.

En 1983, la introducción de la tesis

${ }^{18}$ Deyon, Amiens, 1967, pp. viii-ix. de Jean-Pierre Bardet permite medir el desplazamiento de los problemas.

La existencia de una ciudad constituye un fenómeno prácticamente inexplicable, porque en Europa lo urbano se incluye dentro de la larga duración. En contraste, la dinámica propia de una ciudad puede ser sólo en captar los motores de la reproducción citadina inextricablemente vinculados al modo de producción, implica también calificar y comprender el efecto transformador de la vida urbana. La ciudad es objeto y sujeto. Construida por los hombres, es la plataforma de sus actividades. Al mismo tiempo, los hombres y las mujeres que conforman la ciudad se someten a sus leyes. Ésta determina sus pensamientos, sus comportamientos y una ecología que a su vez participa de las modalidades de la renovación urbana. Lo que quisiera aclarar es este complejo intercambio. ${ }^{19}$

La ciudad adquiere así una doble autonomía. Está ubicada en el centro de las siguientes interrogantes: ¿cuáles son los motores de la urbanización?, ¿cuáles son las capacidades transformadoras de lo urbano? En el plano del conocimiento la ciudad, como tal, se ha convertido en objeto de la historia. Esta especificidad del modo de aprehensión duplica la de lo real y encuentra en ella su justificación. Además de objeto -construido por los hombres y analizado por la ciencia histórica-, la ciudad es también sujeto de la historia. Es más que un marco, más que la simple suma de elementos que la componen. Por su misma existencia, es crea-

19 Bardet, Rouen, 1983, pp. 18-19. 
dora de innovaciones que afectan su propio devenir.

Es fácil fechar el cambio que transforma la posición de la ciudad de subproducto de la investigación, que obedece a otras preocupaciones temáticas, en personaje central. Un artículo de 1974, cuya ambición sobrepasaba la sola dimensión historiográfica confesada, y la tesis de Jean-Claude Perrot, terminada en 1973 y publicada en 1975, constituyen los textos centrales. ${ }^{20} \mathrm{Al}$ afirmar que el sujeto de la historia urbana existe y que hay una unidad y una creatividad de los fenómenos citadinos, estos textos fundaban al mismo tiempo un dominio de investigaciones específicas.

\section{Una experiencia ejemplar}

Un estudio más preciso del libro de Jean-Claude Perrot permitirá despejar mejor sus caracteristicas y dificultades. A parecer si consultamos primero los títulos de los capítulos, el libro se incluye en una de las corrientes dominantes de la historiografía francesa, colocada desde el inicio de los años cincuenta bajo la influencia de Ernest Labrousse, que establece una historia cuantitativa atenta a extraer los movimientos alternados de la coyuntura, las estructuras económicas y sociales de larga duración. Sin embargo, a diferencia de todas las monografías sobre historia urbana del momento, el libro no incluye ningún estudio particular de las estructuras sociales. La crítica

20 Bergeron, "Ville", 1974, pp. 827-876. Perrot, Genèse, 1975. del debate sobre la naturaleza de las sociedades del antiguo régimen, que Jean-Claude Perrot había publicado algunos años antes, conducía evidentemente a renunciar a ello. Pero la solución que adopta en 1975 es distinta de la que sugeriría siete años antes. En 1968 , al considerar que el análisis de las estructuras era necesariamente tautológico, postulaba estudiar las relaciones sociales. Las ceremonias públicas, las formas de asociación, los lugares de encuentro, las manifestaciones de violencia constituían otras tantas dimensiones de una sociabilidad citadina cuya descripción permitía acceder al conocimiento de las sociedades del pasado. El barrio, la calle, el taller, el cabaret constituían los lugares donde se revelaban las formas de es. tar reunidos que, por lo demás, eran con más frecuencia populares que burguesas o aristocráticas. ${ }^{21}$ Pero precisamente todos estos lugares no constituían todavía más que un marco, observatorios privilegiados que favorecían al mismo tiempo el reencuetro y su control.

En 1975, Jean-Claude Perrot considera reduccionista la actitud consistente en creer que se ha comprendido una sociedad después de haber descrito los grupos que la conforman y sus relaciones.

Una lectura perspicaz debe percibir que los comportamientos de la población, la práctica médica, los procesos que regulan la producción, los intercambios, el acondicionamiento de los barrios des-

\footnotetext{
21 Farge, "Treatres", 1979, pp. 984-1015 Roche, Peuple, 1986.
} 


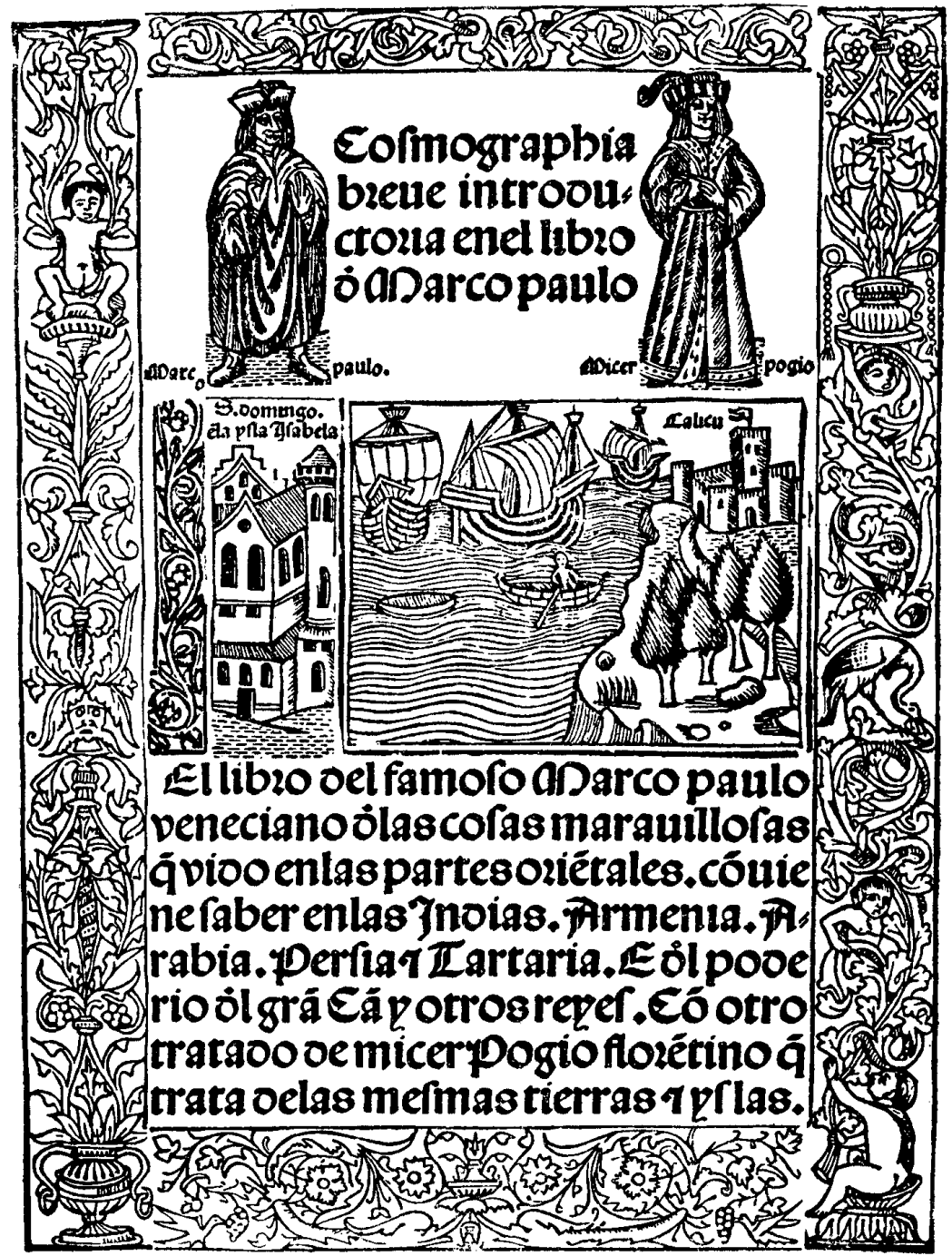


criben con eficacia los fundamentos de la historia social (p. 944).

Así, la ciudad no tiene carácter propio; depende de lo social y es como una escoria (p. 947) suya, como un residuo procedente del enfriamiento superficial de las corrientes de lava, en el sentido en que lo entienden los vulcanólogos. De ahí surge una consecuencia metodológica. La simple yuxtaposición de diversos estudios (demografía, economía, sociedad, cultura) conduciría a hacer caer en ruinas al sujeto: "El primer camino consistiría entonces en cruzar las fuentes entre sí, puesto que se admite que hablan de lo mismo" (p. 947), es decir de la ciudad moderna. De este modo, el historiador no puede confundir las estructuras de la realidad pasada con el recorte de las series de archivos; la primacía de la evidencia del archivo ha sido completamente modificada en beneficio de la identificación y de la construcción de un objeto.

En este sentido el libro es un modelo, una copia teórica, es decir una representación simplificada y abstracta de la realidad que examina, construida a partir de un sistema de relaciones que responde a principios de coherencia interna y que no entra en contradicción con los datos empíricos disponibles. El primer imperativo de la investigación al desarrollarse es pues establecer que su objeto existe en el momento en que lo estudia. Caen, en el siglo XVIr, se convierte en una ciudad moderna, es decir, y para retomar la definición del diccionario, pertenece al tiempo del que habla el historiador del siglo $\mathrm{xx}$. Dos rasgos definen aquí la modernidad urbana: el pasaje de una economía estática a una economía en movimiento, por una parte, y la invención del funcionalismo, por la otra. El primer rasgo lleva a dejar de reducir el cambio a su medida cuantitativa, como lo hace demasiado a menudo la historia seriada.

Las dimensiones de Caen, su producto global, si fueran más conocidos, permanecerian mudos acerca de esta revolución silenciosa que eleva a esta ciudad al grado de urbanización en que se encuentran ya, después de París, cuatro grandes puertos $y$ algunos lugares del interior. (p. 951).

El segundo rasgo conduce a mostrar, en el pensamiento y en la práctica de los habitantes de la ciudad, a partir de la mitad del siglo XVII, la serie de principios vinculados que definen el funcionalismo: para comprenderlo, el sistema socioeconómico debe descomponerse en distintas funciones; entre cada una de estas funciones existen relaciones, covariaciones (riqueza y mortalidad, nivel de población y de comercio, enfermedades y actividades profesionales); y hay entre cada una de las funciones y los espacios en que se realizan una relación simple (lo que justifica desde luego la intervención en el espacio para producir una modificación de la función). Uno y otro rasgos llevan a descalificar el vínculo establecido anteriormente entre la industrialización y la urbanización, entre la proletarización del siglo XIX y los problemas urbanos: "La invención del problema urbano supera por un siglo la del problema social" (p. 9). 
La modernidad del objeto conlleva dos consecuencias. Conduce en primer lugar a otorgar una atención particular a las representaciones que el pasado tiene de sí mismo. El discurso que una sociedad tiene sobre ella misma o el que sus prácticas revelan, informa a la vez del mundo en que vive (en el primer sentido en francés del verbo informar, informer: dar al mismo tiempo un significado y una forma) y al historiador que la estudia (en el segundo sentido de informar: poner al corriente). Sin embargo, nuestra experiencia cotidiana nos advirte: las sociedades son en parte ciegas a su propia naturaleza y multiplican las artimañas interpretativas.

Pero las prácticas que se derivan de esta ceguera parcial -ya se trate de comportamientos demográficos, de la organización del territorio o de los conflictos entre las corporaciones de oficios- tienen su propia eficacia,y siempre es posible considerar las proposiciones de los observadores del pasado como un conjunto de hipótesis a confrontar con los datos empíricos. Segunda consecuencia de la modernidad de la ciudad del siglo xvil: el investigador está instalado en una considerable proximidad con respecto a su objeto. En apariencia se trata de una ventaja: si es relativamente fácil encontrar, en la documentación del siglo XVIII, respuestas a las preguntas que el historiador del siglo $\mathrm{xx}$ plantea, ello es en principio porque las preguntas de ambas épocas a propósito de la ciudad son las mismas. Pero esta facilidad es una trampa; la proximidad vuelve difícil, si no imposible, la crítica de un análisis funcional que, por sí mismo, forma parte de las ideologías; esto impregna toda una parte del análisis urbano de hoy. "Conocer lo borroso de nuestros propios lentes" (p. 11), garantizar un "continuo ir y venir entre las técnicas actuales y el horizonte de las Ilustración" (p. 13), aprovechar la distancia temporal y la densidad hermenéutica que nos separan del siglo XVII; éstos son los medios empleados para organizar una tensión creadora de sentido y comprensiva entre la ciudad de ayer y las categorias que la hacen visible.

Pero al afirmar que "todo el sentido está en la relación entre los distintos niveles" de la organización social, Jean Claude Perrot daba a los historiadores de las ciudades un objetivo particularmente intimidante. ${ }^{22} \mathrm{Si}$ la comprensión aislada de los distintos niveles de la realidad parece no oponer más que problemas tradicionales, vinculados a la insuficiencia de las fuentes, ¿cómo entender las relaciones entre tales niveles? ¿Cómo estudiar el fenómeno sociocultural total donde abundan los riesgos de encontrar casualidades circulares? El grado de complejidad en el que estaba situada la ambición historiográfica implicaba en sí mismo riesgos para la tarea. Dos frases que definen el carácter de la nueva historia urbana, pueden ser reveladoras: "La ciudad se convierte en el personaje central de toda una generación de trabajo", escribían en 1974 L. Bergeron y M. Roncayolo. En 1983 F. Béarida subrayaba: "Los múltiples campos de investigación tienen un gran factor en común: el re-

22 Perrot, Gendse, 1975, p. 947. 
chazo a considerar la ciudad como meramente inerte, sin vida." 23 Ya se ha puesto énfasis en la ambigüedad de las metáforas biológicas en el campo social y no se trata aquí de instruir un mal proceso. El objetivo de la nueva historia urbana no era el de dotar a la ciudad de una casi-intencionalidad, sino de subrayar a la vez la autonomía de lo urbano y su extraordinaria complicación.

Claramente se observan los peligros que implica semejante globalización. Por una parte, muy pocas cosas, incluso en la época preindustrial, no atañen de un modo o de otro a la ciudad. Si nada de cuanto es urbano escapa a la historia urbana, la dilatación y la diversidad del campo de investigación se vuelven extremos. Al mismo tiempo, ¿cómo creer que se puedan englobar fácilmente en una red de definiciones el conjunto de hilos que forman el tejido urbano? A falta de una reflexión suficiente sobre el carácter de dichos vínculos y sobre las mediaciones que autorizan a pasar de un nivel a otro de la realidad, o de un inventario de fenómenos acumulativos y de efectos de bloqueos provenientes de la ciudad, existe el riesgo de producir un discurso cuya función esencial consistiría en conservar la unidad artificial de un espacio de investigación infinitamente extendido. Pero a la inversa, si el espacio citadino no es más que la simple proyección de una sociedad sobre el mapa, la simple traducción de las desigualdades sociales en el terreno, la ciudad es tan sólo una som-

23 Bergeron, "Ville", 1974, p. 827. Bedarida, "French", 1983, pp. 395-406. bra de la caverna. Si el análisis de las relaciones sociales se reduce a mostrar la manera como los hombres se encuentran en las ciudades, entonces la historia urbana no es más que un velo, destinado a enmascarar la incapacidad provisional de la historia social para proporcionar explicaciones sobre las solidaridades y los conflictos que emanen de la construcción misma de las sociedades, y la ciudad, una vez más, es única y solamente un teatro.

\section{Cambios de escala}

Construir claramente la historia urbana como historia problema es sin duda el medio de evitar los dos riesgos simétricos: la complejidad de un modo opacante y la de desvanecer el objeto. Supone explicitar los problemas que tengan por objetivo delimitar las especificidades de lo urbano. Supone, para retomar el título de un artículo con ambiciones más estrechamente delimitadas, comprender "las ciudades como sistemas en un sistema de ciudades". ${ }^{24}$ Según el sentido que se atribuya a la palabra sistema, la investigación puede orientarse en distintas direcciones. Si se define el sistema urbano como el que organiza en una configuración espacial y jerárquica un conjunto de ciudades, entonces se trata de un estudio de las redes y armazones, entendidas en su relación con el espacio. Si se define a la ciudad como "una formación real que se inscribe en un terreno y dentro de una topografía particular, que

\footnotetext{
24 Berry, "Cities", 1964, pp. 147-163.
} 
se imprime en una sociedad estructurada, que se explica por instituciones y se traduce en un género de vida y una cultura". ${ }^{25}$ Entonces la investigación se desarrolla como un análisis sistémico de la formación considerada. En ambos casos, pero en sentidos diferentes, se trata de un cambio de escala en relación con las monografias de ciudades que han servido de marco a la primera renovación de la historia urbana. Me gustaría sugerirlo en dos ejemplos.

Fernand Braudel lo subrayaba desde mediados de los años sesenta: nunca una ciudad se presenta sin el acompanamiento de otras ciudades, más grandes o más pequeñas, cercanas o lejanas, y puntos de apoyo, relevos o rivales. ${ }^{26}$ En una economía preindustrial, la existencia de sistemas urbanos es una hipótesis aceptable. Una ciudad no puede concebirse sin la existencia de un espacio que la englobe o de donde tome lo esencial para su subsistencia (ni los granos, ni la madera, que son productos pesados, viajan, salvo excepciones, a través de largas distancias), una buena parte de su población (los balances demográficos naturales de las ciudades son negativos) y de sus ingresos (puesto que los habitantes más ricos de las ciudades son propietarios de bienes raíces). Pero si las ciudades no mantuvieran ninguna relación entre sí, no serían menos dependientes unas de otras: la ausencia de una revolución agrícola antes del siglo XIX se opone a que la extensión

25 Chevalier, Bones, 1982, cit. p.11.

26 Braudel, Civilisation, 1979, 3 vols. El primer volumen, "Les structures du quotidien", retoma y enriquece un libro publicado en 1967 con un título diferente. de las posibles reducciones en alimentos, en rentas y en hombres sea indefinida. La localización, la dimensión y el ritmo del crecimiento de cada ciudad depende también de los correspondientes a las demás. Plantear un problema de historia urbana es preguntarse cómo tal sistema origina su propia evolución.

Si se considera, por ejemplo, la Francia de alrededor de 1830, antes del desarrollo de una industrialización de nuevo tipo, numerosos indices (evolución de la geografía de los precios o modalidades de la difusión de las innovaciones) presentan un funcionamiento regional distinto al del sistema urbano. ${ }^{27}$ En el norte, la unificación del espacio económico, la intensidad de los vínculos interurbanos, la receptividad de las sociedades citadinas caracterizan su funcionamiento del sistema urbano como red. En el sur, la persistencia del agrupamiento económico existente, el retraso para innovar, la menor intensidad de los flujos de relación, el repliegue más importante de las sociedades urbanas sobre sí mismas, determinan un funcionamiento del sistema urbano como armazón que yux. tapone células (formadas cada una por una modesta metrópoli local y sus tierras interiores) poco relacionadas entre sí. Una primera explicación remite esta diferencia a la historia de la instalación de la red de carreteras que se inició en los años de 1740 y que benefició a más de la mitad norte del país que a la mitad sur. ${ }^{28}$ En las carac-

27 Lepetit, Villes, 1988.

28 Arbellot, "Grande", 1973, pp. 765-791; 
terísticas de la construcción de las rutas se demuestra la influencia del sistema urbano. Bajo el antiguo régimen las rutas construidas reforzaron la armazón urbana más antigua, acentuaron la importancia de París (y de la cuenca parisina) y comunicaron prioritariamente a las ciudades caracterizadas al mismo tiempo por el ejercicio de la función administrativa y por su riqueza en bienes raíces. La misma reproducción de una estructura por otra vuelve a encontrarse después de la revolución. En 1811, la administración de puentes y calzadas estableció la instalación de las rutas imperiales; esta instalación fue también un programa de equipamiento, pues la red no estaba concluida en ese año y la instalación de una ruta determinaba la im. portancia de los financiamientos que recibía. Calcó a su vez la red de las rutas sobre la nueva armazón administrativa del país: de iguales dimensiones, las nuevas cabezas de distrito se beneficiaron de mejores servicios de comunicación que las otras ciudades.

¿Puede decirse entonces que el sistema urbano de los años de 1830 tuvo su origen en la creación de los departamentos y luego en la elección de prefecturas y subprefecturas que formaron su centro? La realidad es más compleja. Pese a una movilización muy activa de las ciudades en el momento de la reforma, ${ }^{29}$ lo que remite desde luego a los equilibrios anteriores a 1789 , el nuevo mapa administrativo se inscribe como ruptura en

Leperit, Chemins, 1984; Arbellot, Atlas, 1987, t.1: "Routes et communications".

29 Ozouf-Marignier, Formation, 1989. relación con el antiguo régimen: las ciudades promovidas o rebajadas en su categoría por el orden administrativo fueron numerosas. En el orden jerárquico, el nuevo equilibrio entre las ciudades, surgido al inicio de la revolución y que favoreció a las ciudades pequeñas y medianas en detrimento de las metrópolis regionales, permanece estructurado así por mucho tiempo: hasta la segunda guerra mundial, Francia sigue siendo un país de ciudades pequeñas. En el orden espacial, la armazón de las cabeceras de distrito desarrolla en todo el territorio una malla uniformadora $y$ tiende a fosilizar esta organización regular. Pero en el orden económico, la reforma administrativa generaliza los antiguos funcionamientos; cada cabecera se convierte en un punto de reunión para los rentistas de la tierra y para los miembros de profesiones liberales (notarios, abogados, médicos) que desarrollan en sus comportamien. tos, sus modos de vida y sus hábitos de consumo, modelos propios de los medios privilegiados (nobleza y alto clero) del siglo xviI. De este modo confieren a dichas ciudades algunas de sus funciones económicas más duraderas al convertirlas en centros de actividades de consumo. ${ }^{30}$

Estas dos nuevas formas de organización del territorio (la carretera y la administración territorial) permiten insistir en la compleja manera como el sistema urbano participa en su propio destino, del que es al mismo tiempo, para emplear el vocabulario estadístico, una variable explicativa y

30 Lepetit, "Event", 1990, pp. 17-37. 
una variable explicada. Esta complejididad obedece a que en el espacio las formas geográficas, los equipos estructurantes y los funcionamientos económicos no coinciden nunca de manera exacta, debido a que lo antiguo y lo nuevo no se encadenan jamás en forma lineal. La carretera, equipamiento estructurante nuevo, refuerza las antiguas configuraciones geográficas al comunicar primero y de mejor manera a las ciudades más importantes. Pero al mismo tiempo subvierte esta geografía al dotar de un nuevo conteniclo relacional a las anteriores formas de organización del territorio y al permitir un funcionamiento más progresista del espacio económico. El mapa aclministrativo establecido bajo la revolución era igualmente una estructura nueva, y se inscribió como ruptura en relación con los mapas antiguos al establecer nuevas jerarquías y proyectar sobre el territorio nuevas distribuciones espaciales. Pero eran asimismo portadoras de modos antiguos de funcionamiento económico de los sistemas urbanos (un conjunto de minúsculas capitales locales) y contribuyeron a garantizar su difusión en el espacio francés y a su persistencia en el tiempo, más allá incluso de la revolución industrial. De este modo, al producir algo nuevo unido a lo viejo y algo viejo unido a lo nuevo, el 'sistema urbano está constantemente en un desequilibrio parcial. En todo momento está comprometido en un proceso histórico de transformación de sí mismo.

Por ello, su evolución no es ni mecánica ni fácilmente previsible. Las nuevas formas de estructuración del espacio se desarrollan de acuerdo con distintas temporalidades. Por otra parte, se aplican a regiones cuyo pasado difiere. En Francia, los sitemas de vivienda y las formas de urbanización son la imagen poco deformada de esquemas muy antiguos, sin duda más que milenarios, de asentamiento. La desigual densidad de las redes administrativas del antiguo régimen y la dominación parisiense son producto de la historia de la construcción del Estado desde la edad media. Las formas de las redes comerciales, la densidad relativa de las ferias episódicas, de los mercados quincenales y de las tiendas permanentes remite sin duda a las dos temporalidades precedentes. Como lo han sugerido los últimos libros de Braudel, las antiguas geografías, "esas realidades amontonadas unas sobre las otras", son constantemente retomadas por los hombres a diferentes escalas en las nuevas construcciones del espacio. ${ }^{31}$

Cambiemos ahora de escala para considerar las sociedades urbanas. Como se sabe, son sociedades mezcladas. El crecimiento, e incluso simplemente la conservación de su nivel de población, supone la existencia de flujos migratorios constantes. La importancia de la cantidad de recién llegados y la extensión de la cuenca migratoria donde se encuentran, dependen del tamaño de la ciudad considerada, de su función y de la coyuntura económica. Orientados en sentido contrario, los flujos de salida siempre son importantes, aun cuando sean más difíciles de captar. Pero,

31 Braudel, L' identité, 1986. 


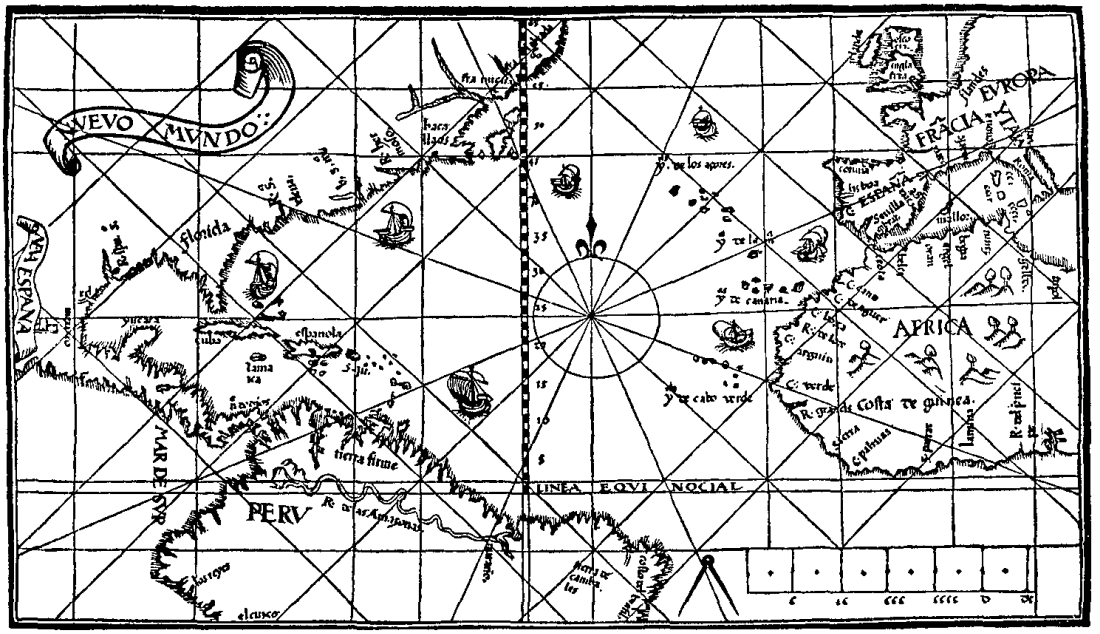

como quiera que sea, la ciudad es una vasta encrucijada donde se mezclan poblaciones estables y poblaciones móviles en los recorridos y en diversos proyectos. Incluso sin existir ninguna otra causa, por simple necesidad demográfica, las sociedades urbanas son sociedades plurales donde el problema de las identidades y las identificaciones se plantea de forma diferente que en las sociedades aldeanas más arraigadas. Tradicionalmente, la historiografia abordaba el problema de dos maneras. Por una parte, como se ha visto, proyectaba sobre las socie. dades urbanas del pasado categorías analíticas que en sí mismas encontraban su fin y su comienzo. La historia podría utilizar las casillas y la retórica de una sociología clasificadora ${ }^{32}$ o 32 Para un ejemplo: Sewell, Structure, 1982; bien optar por la interpretación del lenguaje de los actores, un camino hermenéutico tomado de la antropología cultural que se inspira en Clifford Geertz. ${ }^{33}$ Este autor podía preferir las clases, los órdenes o las categorias socioprofesionales como principio explicativo de las sociedades antiguas. En todos los casos, la predeterminación del método y de la clasificación resolvía de un solo golpe el pro. blema de las identidades sociales. Por otra parte, la historiografia tradicional tendía a considerar las poblaciones es-

para una crítica: Gribaudi, "Catégories", 1990 , pp. 1365-1402, y de manera más general, el conjunto temático de Annales titulado "Mobilites", noviembre-diciembre de 1990.

33 Para un ejemplo: Darnton, Great, 1984; para una crítica: Chartier, "Text", 1985, pp. 682695 y Levi, "Pericoli", 1985, pp. 269-277. 
tables de las ciudades como las únicas portadoras de una identidad citadina, preservada por medidas de control activo del flujo de inmigrantes y por una política de integración muy restrictiva, y aplicaba a las poblaciones migrantes el concepto de aculturación. ${ }^{34}$ Esto significaba omitir la variedad de las trayectorias y los proyectos individuales o familiares, la multiplicidad de las estructuras de posible identificación, la complejidad de la coyuntura o la de las situaciones donde se actualizan las diversas identidades de las poblaciones urbanas. ${ }^{35}$

Recordamos las primeras palabras del prefacio del gran libro de E.P. Thompson, The making of the english working class:

Este libro tiene un título torpe, pero cumple con su propósito. Hablo de "hechura" porque es un estudio en un proceso activo, que le debe tanto al medio como a las condiciones. La clase obrera no surgí como el sol en un momento determinado. Estaba presente en su propia hechura. ${ }^{36}$

En un enfoque similar, una nueva historia social de las ciudades, en vez de considerar como meros datos los grupos y, por experiencia, la pertenencia a ellos, que abarcan y definen a los individuos, intenta invertir

3. Para un ejemplo y escenificación de un vaivén en detrimento de las poblaciones urbanas arraigadas, Chevalier, Classes, 1958.

35 Para una demostración particularmente lograda, Gribaudi, Itinératres, 1987.

36 Thompson, Making, 1963, prefacio. Significativamente, la primera traducción francesa de este libro data de 1988. la perspectiva. Se pregunta acerca de la manera en que las relaciones de múltiples niveles, establecidas en todos sentidos en las sociedades citadinas, crean solidaridades, esbozan redes, definen identidades. El verdadero problema, escribe Simona Cerutti en el libro hasta el momento más representativo de este nuevo enfoque,

es comprender cómo individuos con historias y experiencias diferentes pueden decidir reunirse y, todavía más, reconocerse a través de una identidad social común. En resumen, se trata de interrogarse sobre la relación entre la racionalidad individual y la identidad colectiva. ${ }^{37}$

La lista de referencias posibles para la constitución de las identidades citadinas es larga. Se puede citar, sin orden, el sexo, el grupo de edad, la familia, la región (para los migrantes internos), el barrio, el oficio, la cofradía, la orden, las instituciones de poder citadino (civil, religioso y en ocasiones militar), la religión, etcétera. No se trata de elegir entre estas distintas clasificaciones para pretender que alguna de ellas (o la combinación de varias) constituya la verdad de las sociedades preindustriales. Se trata de imaginarlas como un conjunto de recur. sos que pertenecen al ser mismo de la ciudad en un momento y en un lugar determinados, definiendo el espacio de acción de los habitantes de la ciudad. Este espacio no es evidentemente el mismo para todos: los recursos movilizantes dependen de los lu-

37 Cerutti, ville, 1990, cit. p. 14. 
gares iniciales de inserción social, de las trayectorias familiares $e$ individuales, de la coyuntura del momento, de las capacidades de interpretación de la realidad cotidiana.

Al inicio del siglo XviII, un inmigrante comerciante de seda, en Turín y originario del otro lado de los Alpes, puede ser miembro de una corporación de oficio, de una cofradía religiosa, de la administración municipal o bien cumplir varios de estos papeles al mismo tiempo. Sus redes familiares de alianza pueden reforzar esos primeros vínculos $o$, por el contrario, establecer otros con base en una complementariedad de la actividad económica, del origen $o$ incluso de la residencia. La combinación de sus elecciones, impuestas o razonadas, cletermina al mismo tiempo la importancia de sus recursos, las características de su identidad social y el carácter de la estratificación social del momento basada en los principios alternativos de la cohesión o de la homogeneidad. Así, tan sólo tardíamente, en los años de 1730 aparecen en Turin nuevas identidades sociales tomadas del lenguaje del trabajo, convirtiéndose el oficio en un criterio decisivo de estratificación y de representación sociales (las primeras corporaciones se fundaron 50 años antes).

En contra de un enfoque objetivista, que haría de los grupos sociales categorías naturales, reconocibles mediante series de indicadores transmisibles, se desarrolla, como se observa, un enfoque más subjetivo, más individualizante, más interesado por las redes, las estrategias, las situaciones, los procesos que por las estructuras que se consideran estables. El problema, entonces, consiste en las modalidades y los efectos del recubrimiento parcial, del recorte y de las exclusiones, de los diferentes elementos que forman la paleta de la identidad. El problema es su actualización por los actores sociales, en los procesos de identificación recíproca que establece una sociedad urbana en constante redefinición, y que se encuentra establecida por ellas.

De ahí nace una nueva historia local de la política. Las instituciones municipales pertenecen a la gama de recursos inequitativamente movilizados por los habitantes de la ciudad, pues no puede escribirse una historia social de la ciudad sin tomar en cuenta la historia social de las instituciones. La organización institucional de las ciudades del norte de Francia y del sur de los Países Bajos españoles, por ejemplo, es primero una construcción sociocultural: gobierno de las elites, autonomía local, dispositivo de economía social (welfare town, si puede decirse), todo ello impregnado de valores católicos, y que definen un "modelo hispano-tridentino de la buena ciudad". ${ }^{38}$ Las instituciones urbanas se inscriben al fin del antiguo régimen en una historia secular, pero la coyuntura del momento es lo que les da sentido y explica las configuraciones particulares que adquieren en el espacio social. Las modalidades de articulación de las formas y de sus usos no son sólo un problema que dependa de la urbanización y del espacio físico de la ciudad, sino también del derecho y de

38 Guignet, Pouvoir, 1990. 
las instituciones. A su vez, el rechazo violento del orden urbano debe analizarse de esta manera; no sólo como la manera de revelar comportamientos, convicciones, relaciones, valores, que de otro modo seguirían siendo opacos; no sólo como la oportunidad de restituir un contexto sociopolítico que le dé sentido, sino además como constitutivo de su propio contexto, de las identidacles citadinas y de un saber social urbano. 39

Cuanclo fui invitado en 1984 a esbozar un panorama semejante de la historia urbana para una revista italiana, me permití en mi conclusión un tono pesimista; los riesgos de ver disolverse un objeto demasiado complejo no me parecían desdeñables. ${ }^{40}$ Se comprenderá, me imagino, que actualmente ya no adopto el mismo criterio. Existen trabajos que responden al vasto programa que indicaba yo entonces, es decir el de reconocer la diversidad de las formaciones humanas que se suceden tras la aparente organización de los lugares y del vocabulario, para así contribuir a una definición histórica de lo urbano. Hay investigaciones que, en vez de reducir la complejidad de la ciudad, se esfuerzan por utilizarla para comprender los procesos mediante los cuales los actores vuclven a definir de manera constante y al mismo tiempo consciente e involuntaria, la organización de lo social entendido éste en el sentido más amplio. Más que ninguna otra, quizá la

39 Farge y Revel, Logiques, 1988. Descimon, "Barricades", 1990, pp. 397-422.

40 Lepetit, "Storia", 1984, pp. 639-666. En este lugar se retoman elementos de la primera parte de este texto. historia urbana proporciona la oportunidad de acabar con la tautología, con las descripciones en términos de categorías predeterminadas.

\section{BIBLIOGRAFIA}

-Arbellot, B., "La grande mutation des routes de France au milieu du XviI ${ }^{\mathrm{e}}$ siècle", $A$ n nales, Economies, Sociétés, Civilisations, 1973.

-Arbellot, G., B. Lepetit y J. Bertrand, Atlas de la Révolution françatse, t. 1: "Routes et communications", París, 1987.

-Bardet, J.P., Rouen aux XVIf et XVII siècles, les mutations d'un espace social, París, 1983.

-Bedarida, F., "The french approach to urban history: an assessment of recent methodological trends", en D. Fraser y A. Sutcliffe (comps.), The pursuit of urban bistory, Londres, 1983.

"The growth of urban history in France: some methodological trends", en The study of urban bistory, J. Dyos, Londres, 1968.

-Bergeron, L. y M. Roncayolo, "De la ville préindustrialle à la ville industrielle: essai sur l'historiographie française", Quaderni Storici, 1974.

-Berry B., "Cities as systems within systems of cities", Papers of Regional Science Association, núm. 13, 1964.

-Biget, J.L., J.C. Herve y Y. Thebert, Les cadastres anciens del villes et leur trattement par l'informatique, Roma, 1989.

-Bloch, M., L'étrange défaite, París, 1946 (reedición París, 1990).

-Boudon, F., A. Chastel, H. Couzy y F. Hamon, Système de l'architecture urbaine. Le quartier des Halles à París, París, 1977.

-Braudel, F., Civilisation matérielle. Economie et capitalisme $x v^{e}-x v I I^{e}$ siècle, 3 vols., París, 1979. Hay traducción al castellano: Civilización material. Economía 
$y$ capitalismo, siglos $x v \cdot x V I I$, Alianza Editorial, Madrid, 1984.

vols., París, 1986.

Carriere, F. y P. Pinchemel, Le fait urbain en France, París, 1963.

-Castells, M., La question urbaine, París, 1972. Hay traducción al castellano: La cuestión urbana, Siglo XXI México, 1974.

Castex, J., P., Celeste y P. Panerai, Lecture d'une ville: Versailles, París, 1980.

-Cerutti, S., La ville et les métiers. Natssance d'un langage corporatif (Turin $\left.17^{*}-18^{\circ}\right)$, París, 1990.

-Chartier, R., "Text symbol and frenchness", Journal of Modern History', 1985.

-Chevalier, B., Les bones villes de France du X $\|^{e}$ siècle au Xvf siècle, París, 1982.

-Chevalier, L., Classes laborieuses et classes dangereuses à París pendant la première moitié du XIX siècle, Plon, París, 1958.

-Choay, F, L'urbanisme, utopies et réalités, Paris, 1965.

-Darnton, R., The great cat massacre and other episode in french cultural bistory, Nueva York, 1984. Hay traducción al castellano: La gran matanza de gatos $y$ otros episodios de la cultura francesa, Fondo de Cultura Económica, México, 1987.

-Demangeon, A. y B. Fortier, Les vaisseaux et les villes. L'arsenal de Cherbourg, Bruselas, 1978.

-Descimon, R., "Les barricades de la Fronde parisienne. Une lecture sociologique", Annales Economies, Sociétés, Civilisations, núm. 2, 1990.

-Deyon, P, Amiens capitale provinciale, étude sur la société urbaine au Xvilf siècle, París, 1967.

-Duby, G., Histoire de la France urbaine, 5 vols., Seuil, París.

-Dugrand, R., Villes et campagnes en Languedoc, París, 1963.

-Farge, A., Vivre dans la rue à Paris au $x V I /{ }^{e}$ siècle, París, 1979
-Farge, A. y A. Zysber, "Les tréatres de la violence à Paris au XVII ${ }^{\mathrm{e}}$ siècle", Annales Economies, Sociétés Civilisations, núm. 4, 1979.

-Farge, A. y J. Revel. Logiques de la foule. L'affaire des enlèvements d'enfants, París, 1750 (París, 1988).

-Foucault, M., Les machines à guèrir, aux origines de l'bopital moderne, París, 1976.

, Surviller et punir, París, 1975. Hay traducción al castellano: Vigilar y castigar, siglo XXI Editores, Madrid, 1988.

-Furet, F, Latelier de l'bistoire, París, 1982.

-Garden, M., Lyon et les byonnats au $x v i r^{\mathbf{e}}$ siècle, Pacís. 1970.

-Garden, M., y Y. Lequin, Costruire la ville, $x v i f^{e}-x X^{e}$ siécles, Lyon, 1984.

-Goubert, P., Beauvais et le beauvaisis de 1600 a 1730 , Panís, 1960

-Gribaudi, M., Itinéraires ouvriers. Espaces et groupes sociaux; á Turin au début du XIX $\mathrm{X}^{\mathrm{e}}$ siècle, Paris, 1987.

-Gribaudi, M. y A. Blum, "Des catégories aux liens individuels: l'annalyse statisque de l'espace social", Annales Economies, Sociétés Civilisations, núm. 6, 1990.

-Guignet, Ph., Le pouvoir dans la ville au XVII siècle. Pratiques politiques, mobilité et éthique sociale de part er d'autre de la frontière franco-belge, París, 1990.

"Histoire et urbanisation", Annales Economies, Sociétés Civilisations, 1970, núm. 4.

-Jacquemet, G., Belleville au XDx siècle, du faubourg à la ville, París 1984.

-Lefebvre, H., La penseé marxiste et la ville, París, 1972. Hay traducción al castellano: El pensamiento marxista y la ciudad, Extemporáneos, México, 1973.

- La révolution urbaine, París, 1970. Hay traducción al castellano: La revolución urbana, Alianza Editorial, Madrid, 1972 (Alianza de bolsillo, 278). 
-Le Goff, J., La nouvelle bistoire, París, 1978.

-Lepetit, B. y J. Hook. La ville et l'innovation. Relais et réseaux de diffusion en Europe, 14e. $19^{\circ}$ siècles, París, 1987.

-Lepetit, B., Cbemins de terre er voies d'eau: néseaux de transport er onganisation de l'espace en France, 1740-1840, París, 1984. revolution and the french urban system";, Journal of Historical Geograpby, vol. Xv, núm. 1, 1990.

___ _ " La storia urbana in Francia, scenografia di uno spazio di ricerca", Societá e Storia, 1984.

__ _ Les villes dans la France moderne (1740-1840), Albin Michel, París, 1988.

-Levi G., "I pericoli del geertzimo", Quaderni Storici, 1985.

-Livet, G. y B. Vogler (comps.), Poivoir; ville et société en Europe, 1650-1750, París, 1983.

-Lojkine, J., Le marxisme, L'Etat et la question urbaine, París, 1977. Hay traducción al castellano: $E l$ marxismo, el Estado y la cuestión urbana, Siglo XXI Editores, México, 1979.

-Magri, S. y C. Topalov (comps.), Villes ouvrières, 1900-1950, París, 1989.
-Ozouf-Marignier, M.V., La formation des départements. La représentation du territoire français à la fin du Xvit siècle, París, 1989.

-Perrot, J. C., Genèse d'une ville moderne, Caen au Xvife siècle, París, 1975.

_. "Rapports sociaux et villes au XviI" siècle", Annales Economies, Sociétés Cletlisations, 1968.

-Pumain, D. y T. Saint Julien, "Fonctions et hiérarchies des villes françaises. Étude du contenu des classifications réalisées en France entre 1960 et 1974", Annales de Geographie, 1976.

Roche, D., Le peuple de Parts. Essat sur la culture populaire au Xvir $r^{e}$ siècle, París, 1986.

- "Urban history in France", en Urban History Yearbook, 1980.

nouvelle bistoire, París, 1978.

-Sewell, W. H., Structure and mobility: the men and women in Marseille, 1820 1870, Cambridge-París, 1982.

-Thompson, E. P., The making of tbe english working class, Londres, 1963. Hay traducción al castellano: La formación bistórica de la clase obrera: Inglaterra 1780-1932, Laia, Barcelona, 1977. 